\title{
String Structures and the Path Fibration of a Group
}

\author{
A. L. Carey $^{1}$ and M. K. Murray ${ }^{2}$ \\ 1 Department of Pure Mathematics, University of Adelaide, GPO Box 498, Adelaide SA 5001, \\ Australia \\ ${ }_{2}$ Mathematics Research Section, School of Mathematical Sciences, Institute of Advanced Studies, \\ The Australian National University, GPO Box 4, Canberra ACT 2601, Australia
}

Received November 20, 1989; in revised form February 11, 1991

\begin{abstract}
We use the realisation of the universal bundle for the loop group as the path fibration of the group to investigate the string class, that is the obstruction to a loop group bundle lifting to a Kac-Moody group bundle. In the case that the loop group bundle is constructed by taking loops into a principal bundle we show that the classifying map is the holonomy around loops and give an explicit formula for the string class relating it to the Pontrjagin class of the principal bundle.
\end{abstract}

\section{Introduction}

Consider a principal bundle $\pi: P \rightarrow M$, with structure group a Lie group $G$, a basepoint $m_{0}$ chosen in $M$ and a basepoint $p_{0} \in \pi^{-1}\left(m_{0}\right)$ chosen in the fibre of $P$ over $m_{0}$. For any space $X$ with basepoint $x_{0}$ we denote by $\Omega X$ the space of smooth loops in $X$ based at $x_{0}$. To be precise, by a smooth loop we mean a smooth path whose endpoints are coincident and for a based loop the endpoints coincide at the basepoint. We take the basepoint of $G$ to be the identity $e$. With these definitions we can construct another principal bundle $p: \Omega P \rightarrow \Omega M$ with structure group $\Omega G$. The map $p$ sends a loop $\gamma(\theta)$ in $P$ to the loop $\pi(\gamma(\theta))$ in $M$. We shall call such a principal $\Omega G$ bundle a loop bundle.

If $G$ is a compact Lie group then the loop group $\Omega G$ has a well-known central extension (see for instance Pressley and Segal 1986)

$$
1 \rightarrow U(1) \rightarrow \widehat{\Omega G} \rightarrow \Omega G \rightarrow 1,
$$

and therefore for any principal $\widehat{\Omega G}$ bundle there is an induced $\Omega G$ bundle obtained, for instance, by composing transition functions for $\widehat{\Omega G}$ with the homomorphism $\Omega G \rightarrow \Omega G$. Given a principal $\Omega G$ bundle then, it is natural to ask when it is induced in this way from an $\widehat{\Omega G}$ bundle. In such a case we say that the $\Omega G$ bundle lifts to an $\widehat{\Omega G}$ bundle. An analogous problem arises for the group $S O(n)$ and the 
extension

$$
1 \rightarrow \mathbf{Z}_{2} \rightarrow \operatorname{Spin}(n) \rightarrow \operatorname{SO}(2 n) \rightarrow 1
$$

An oriented Riemannian manifold has a reduction of its frame bundle to $S O(n)$ and if this reduced frame bundle is induced by some $\operatorname{Spin}(n)$ bundle we say that the manifold is spin.

Similarly in this case a principal $G$ bundle $P \rightarrow M$ is called string if the associated bundle $\Omega P \rightarrow \Omega M$ lifts.

Let us denote by $L X$ the space of (free) smooth loops in $X$. Note that $\Omega X \subset L X$ and that $L G$ is the semi-direct product of $\Omega G$ and $G$. Then all we have just said also works with $\Omega$ replaced by $L$.

It was shown by Killingback (1987) that for the analogous problem for $L G$ bundles, the obstruction to a bundle being string is a class in $H^{3}(L M, \mathbf{Z})$. This class can be defined by considering the central extension (1.1) of groups with $\Omega$ replaced by $L$. From this is constructed a long exact sequence in non-abelian sheaf cohomology,

$$
H^{1}(L M, U(1)) \rightarrow H^{1}(L M, \widehat{L G}) \rightarrow H^{1}(L M, L G) \stackrel{\delta}{\rightarrow} H^{2}(L M, U(1)) .
$$

Recall that, in general, the transition functions of an $H$ bundle define a class in $H^{1}(L M, H)$. So the transition functions of the bundle $L P$ define a class in $H^{1}(L M, L G)$ and the bundle lifts if this class is the image of a class in $H^{1}(L M, \widehat{L G})$ which, by the exactness of the sequence, is precisely when its image under $\delta$ vanishes. This image defines a class in $H^{2}(L M, U(1))$, and using the coboundary map

$$
H^{2}(L M, U(1)) \rightarrow H^{3}(L M, \mathbf{Z})
$$

from the long exact sequence in sheaf cohomology constructed from the short exact sequence

$$
1 \rightarrow \mathbf{Z} \rightarrow U(1) \rightarrow \mathbf{R} \rightarrow 0
$$

this is mapped to an element of $H^{3}(L M, \mathbf{Z})$. Killingback calls this class the string class of the bundle and shows that the string class is obtained from the Pontrjagin class of the bundle $P \rightarrow M$ in $H^{4}(M, \mathrm{Z})$ by pulling back via the evaluation map

$$
\mathrm{ev}: L M \times S^{1} \rightarrow M,
$$

and then integrating over the circle.

The ideas of Killingback can also be applied to the case of based loops, and we shall refer to the corresponding class as the string class although really it is the restriction of the string class in $H^{3}(L M, \mathbf{Z})$ to $H^{3}(\Omega M, \mathbf{Z})$. The methods we have developed here do not seem to readily generalise to $L G$.

This "obstruction theory" approach to characteristic classes is only one of a number of approaches. We will show that for the loop group another standard approach involving classifying spaces takes a particularly simple form. Not only is this useful from the point of view of string structures but it provides a nice concrete example of a theory that in full generality is rather abstract.

In Sect. 2 we recall the definition of the universal principal bundle for a group and the classifying map and their relationship with characteristic classes. These ideas are applied in Sect. 3 to the loop group where we show that the universal 
bundle is the path fibration of the group and that the classifying map is the holonomy. In Sect. 4 these results are used to define the string class of an $\Omega G$ bundle and show that it is the obstruction to it lifting to an $\widehat{\Omega} G$ bundle. Finally in Sect. 5 we connect up with the calculations of Coquereaux and Pilch (1989) and consider the special case of a loop bundle. We calculate the string class and show that it is the same as the integral over the circle of the pullback by the evaluation map of the Pontrjagin form.

\section{Classifying Spaces and Characteristic Classes}

In general the topological properties of principal bundles depend on the following result (see for instance Husemoller 1966). Given a topological group $G$ there is a "universal $G$ bundle" $E G \rightarrow B G$ which is determined (up to homotopy equivalence) by the fact that it is a $G$ bundle and $E G$ is a contractible space. The space $B G$ is called the classifying space. To say this is a universal bundle means that for any principal bundle $P \rightarrow M$ there is a map $f: M \rightarrow B G$, called the classifying map, which is unique up to homotopy, and such that $P$ is isomorphic to the pull back via $f$ of the bundle $E G$.

The fact that $P$ is isomorphic to $f^{*} E G$ is equivalent to the existence of a map $f: P \rightarrow E G$ which preserves the group action and such that the diagram

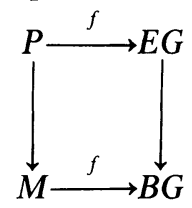

commutes.

Recall that two maps are homotopic if they can be deformed continuously one into the other and that two bundles $P, P^{\prime}$ are homotopy equivalent if there are bundle homomorphisms $f: P \rightarrow P^{\prime}$ and $g: P^{\prime} \rightarrow P$ such that if $f \circ g$ and $g \circ f$ are both homotopic to the respective identity maps. So they are inverses of each other "up to homotopy."

In general $B G$ is an infinite dimensional space and has a rather abstract construction. If $G=\mathbf{Z}$ the universal bundle is

$$
\exp 2 \pi i: \mathbf{R} \rightarrow S^{1}
$$

and if $G=S^{1}$ the classifying space is the space of projective unitary operators on a Hilbert space. An example familiar to physicsts is when $G=\mathscr{G}$, the gauge group of some gauge theory. Then if $\mathscr{A}$ is the space of connections suitably basepointed so that the action of $\mathscr{G}$ is free then

$$
\mathscr{A} \rightarrow \mathscr{A} / \mathscr{G}
$$

is the universal $\mathscr{G}$ bundle (see Singer (1978) for the topology and a proof of the local triviality of (2.3)). The proofs of all these claims follow immediately from the characterisation of the universal bundle as a $G$ bundle with contractible total space.

The classifying space is related closely to the theory of characteristic classes. In general we would like a characteristic class $c$ to assign to any $G$ bundle $P$ over 
a space $M$ an element $c(P)$ of the cohomology $H^{*}(M)$. It has to satisfy the "naturality" property that if $f: N \rightarrow M$ is a map with $f^{*}$ the induced map on cohomology and $f^{*}(P)$ the pull back bundle over $N$ then

$$
c\left(f^{*}(P)\right)=f^{*}(c(P)) .
$$

All characteristic classes arise in the following way. If $\xi$ is a class in $H^{*}(B G)$ then for any bundle $P \rightarrow M$ we can choose a classifying map $f: M \rightarrow B G$ and define

$$
c_{\xi}(P)=f^{*}(\xi) .
$$

Notice that although the classifying map of a bundle is not unique because they are all homotopic the induced map $f^{*}$ on cohomology is unique. So a $G$ bundle over $M$ determines a unique map $H^{*}(B G) \rightarrow H^{*}(M)$. It is straightforward from the uniqueness properties that $c_{\xi}$ is a characteristic class. Moreover given any characteristic class we can take $\xi=c(E G)$ and then

$$
c=c_{\xi} \text {. }
$$

\section{The Classifying Map of the Loop Bundle and the String Class}

We shall assume from now on that the Lie group $G$ is simply connected. In the case of the loop group the universal bundle is remarkably easy to describe. Consider the space $P G$ of all smooth paths in $G$ starting at the identity and the path fibration

$$
P G \rightarrow G
$$

which sends a path to its endpoint. We give the space $P G$ the Fréchet topology and then (3.1) is a principal $\Omega G$ bundle, where $\Omega G$ acts by pointwise multiplication. For these and other questions relating to the correct choice of topology we refer the interested reader to the Appendix.

The space $P G$ is contractible as we can consider the family of maps

$$
F_{\lambda}: P G \rightarrow P G
$$

which send the path $t \mapsto g(t)$ to the path $t \mapsto g(\lambda t)$. This gives a curve joining the identity $\left(F_{1}\right)$ to the constant map $\left(F_{0}\right)$; that is, a contraction. It follows that the path fibration is the universal bundle for loop group bundles. Moreover, as we shall see, in the case of bundles of the form $\Omega P \rightarrow \Omega M$ the classifying map also takes a simple form.

Choose a connection for the bundle $P \rightarrow M$. Then for any loop $\gamma$ in $P$ there is a corresponding unique horizontal path $\gamma_{h}$ beginning at $p_{0}$ with

$$
\pi \circ \gamma=\pi \circ \gamma_{h} \text {, }
$$

namely the horizontal lift beginning at $p_{0}$ of the loop $\pi \circ \gamma$ in $M$. Define the holonomy of $\gamma, \operatorname{hol}(\gamma)$, a path in $G$, by

$$
\gamma=\gamma_{h} \operatorname{hol}(\gamma) .
$$

If $g$ is a loop then $\gamma g$ has the same image in $M$ as $\gamma$ so that $(\gamma g)_{h}=\gamma_{h}$ and therefore $\gamma g=(\gamma g)_{h} \operatorname{hol}(\gamma) g$ from (3.4) and

$$
\operatorname{hol}(\gamma g)=\operatorname{hol}(\gamma) g .
$$


Hence hol: $\Omega P \rightarrow P G$ is a map preserving the group action of $\Omega G$ and therefore descends to a map $\Omega M \rightarrow G$, which we shall denote by the same symbol, to define a commuting diagram as in (2.1),

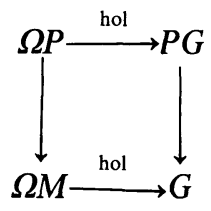

It follows that hol: $\Omega M \rightarrow G$ is the classifying map. This map is the holonomy in the usual sense; given a loop in $M$ we lift it to $P$ horizontally, beginning at $p_{0}$ and the difference between the endpoints is the holonomy.

From the general theory described in Sect. 2 we know that the string class defines an element of $H^{3}(B \Omega G, \mathbf{Z})=H^{3}(G, \mathbf{Z})$ and we shall show that this is the standard generator $\omega$, defined as a $G$ invariant form by its value at the identity

$$
\omega(X, Y, Z)=\frac{1}{8 \pi^{2}}\langle X,[Y, Z]\rangle,
$$

where $\langle$,$\rangle is the Killing form normalised so that the square of the length of the$ longest root is 2 (see for instance, Pressley and Segal (1986) page 49) and $X, Y$ and $Z$ are in the Lie algebra of $\Omega G$.

\section{Constructing the Lift}

Consider the general case where we have an $\Omega G$ bundle $Q$ over a space $X$ and therefore a commuting diagram as in (2.1),

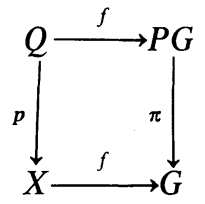

where $f: X \rightarrow G$ is the classifying map. Define the string class $s(Q) \in H^{3}(X, \mathbf{Z})$ to be the cohomology class of the form $f^{*}(\omega)$, where $\omega$ is defined in (3.7). Assume that this class vanishes; we shall show that the bundle $Q$ lifts by giving an explicit construction of the lift $\hat{Q}$. We shall assume from now on that $Q$ is simply connected.

As the string class is zero there is a two form $\mu$ with

$$
d \mu=f^{*}(\omega) \text {. }
$$

If we pull $\omega$ back to $P G$ it is, of course, cohomologous to zero as $P G$ is contractible. If $g$ is a path in $G$, and $X_{1} g$ and $X_{2} g$ to vector fields along the path, or tangent vectors at $g$, then

$$
c\left(g, X_{1} g, X_{2} g\right)=\frac{1}{8 \pi^{2}} \int_{0}^{1}\left(\left\langle X_{1}, d X_{2}\right\rangle-\left\langle X_{2}, d X_{1}\right\rangle\right)
$$

is a right $\Omega G$ invariant 2 form on $P G$ satisfying $d c=\pi^{*}(\omega)$. Moreover restricted 
to any fibre this two form is the standard $\Omega G$ invariant two form $c$ which defines the central extension of the loop group.

Consider now the two form $F$ on $Q$ defined by

$$
F=f^{*} c-p^{*} \mu
$$

which satisfies $d f=0$. We would like $2 \pi i F$ to be the curvature of the $U(1)$ bundle $\hat{Q} \rightarrow Q$ but for that $F$ needs to be an integral form. Let $\alpha_{1}, \ldots, \alpha_{r}$ be cycles generating the two dimensional homology of $X$ and let $\hat{\alpha}_{1}, \ldots, \hat{\alpha}_{r}$ be lifts of these cycles to $Q$, that is $p \circ \hat{\alpha}_{i}=\alpha_{i}$. Notice that the choice we have made of $\mu$ in Eq. (4.2) is arbitrary up to the addition of any $\gamma$ satisfying $d \gamma=0$. Now choose a cohomology class in $H^{2}(X, \mathbf{Z})$, represented by a form $\gamma$, by requiring that

$$
\int_{\alpha_{i}} \gamma=\int_{\hat{\alpha}_{i}} f^{*} c-p^{*} \mu \text {. }
$$

Then if we replace $\mu$ by $\mu-\gamma$ we have that $\int_{\hat{\alpha}_{i}} F=0$ and moreover on restriction to a fibre of $Q \rightarrow X F$ is equal to $f^{*}(c)$ which is integral. As the vertical cycles and the $\hat{\alpha}_{i}$ generate the two dimensional homology of $Q$ it is clear that $F$ is now integral.

Notice that we could have chosen $F$ to have any integral values on the $\hat{\alpha}_{i}$. These choices correspond to different string structures. As $\Omega G$ is a central extension of $\Omega G$ any $U(1)$ bundle on $X$ can be pulled back to $Q$ and "tensored" with an $\widehat{\Omega G}$ bundle by regarding $U(1)$ as being in the center of $\widehat{\Omega G}$. By tensoring here we mean multiplying transition functions, this makes sense, i.e. the cocycle condition for transition functions is preserved, because the transition functions of the $U(1)$ bundle commute with the transition functions of the $\widehat{\Omega G}$ bundle.

Now that we have the 2 form $F$ we will apply a construction analogous to that in Murray (1988) to obtain $\hat{Q}$. Before doing that let us recall the construction of Murray (1988) as we shall need it when constructing $\hat{Q}$. We start with the group $\mathscr{P} \Omega G$ of paths beginning at the identity in $\Omega G$. Then $\mathscr{P} \Omega G \times U(1)$ is made into a group with the product

$$
(f, z)(g, \omega)=(f g, c(f, g) z \omega)
$$

where

$$
c(f, g)=\exp 2 \pi i \int c,
$$

and the integral is over any surface with boundary the triangle in $\Omega G$ with vertices $1,1 g(1)$ and $f(1) g(1)$ and edges $1 g(t), f(t) g(1)$ and $f(t) g(t)$ with orientation chosen so that the edge $1 g(t)$ points in the positive direction.

Let $\Omega \Omega G$ be the subgroup of all loops in $\mathscr{P} \Omega G$. Then the set $K=\left\{\left(g, h^{-1}(g)\right) \mid g \in \Omega \Omega G\right\}$, where hol $(g)$ is the exponential of the integral of $2 \pi i c$ over any two surface with boundary $g$, is a normal subgroup and the Kac-Moody central extension $\widehat{\Omega G}$ is the quotient. The obvious projection map defines the homomorphism $\Omega G \rightarrow \Omega G$. (Note that although the boundary of the triangle is piecewise smooth, we do not need to consider this boundary as a loop in its own right so we do not need to move outside the class of smooth loops.)

Now let $\mathscr{P} Q$ be the space of all smooth paths in $Q$ beginning at a basepoint $q_{0}$ and define an action of $\mathscr{P} \Omega G \times U(1)$ on $\mathscr{P} \mathrm{Q} \times U(1)$ by the product

$$
(q, z)(g, w)=(q g, c(q, g) z w)
$$


where

$$
c(q, g)=\exp 2 \pi i \int F
$$

and the integral is over any surface spanning the triangle in $Q$ with vertices $q_{0}, q_{0} g(1)$ and $q(1) g(1)$ and edges $q_{0} g(t), q(t) g(1)$ and $q(t) g(t)$ oriented so that the edge $q_{0} g(t)$ points in the positive direction. Notice that we need $Q$ simply connected to be sure of finding such a surface and that the integrality of $F$ means that $c(q, g)$ is independent of the surface we choose.

We have to check that with this group product the action of $\mathscr{P} \Omega G \times U(1)$ on $\mathscr{P} Q \times U(1)$ is really an action, that is

$$
(q, z)((f, w)(g, u))=((q, z)(f, \omega))(g, u) .
$$

If one draws all the surfaces of integration one discovers that they form a tetrahedron and the integrality of $F$ after integration over this tetrahedron gives the required result. Now, in general, if we have a manifold $Q$ with an integral closed two form $F$ there is a line bundle over $Q$ with curvature $2 \pi i F$. If $X$ is simply connected there is a standard way of constructing this line bundle using paths. On the space $\mathscr{P} Q \times U(1)$ we define an equivalence relation by saying that $(q, z)$ and $\left(q^{\prime}, z^{\prime}\right)$ are equivalent if $q$ and $q^{\prime}$ have the same endpoint and $c\left(q, q^{\prime}\right) z=z^{\prime}$, where

$$
c\left(q, q^{\prime}\right)=\exp 2 \pi i \int c
$$

and the integral is over the surface with edges $q$ and $q^{\prime}$ oriented so that the edge $q^{\prime}$ points in the positive direction. The set of equivalences classes is denoted by $\hat{Q}$.

It is straightforward to check that

1. The action of $\mathscr{P} \Omega G \times U(1)$ preserves the equivalence relation and therefore acts on $\hat{Q}$.

2. The isotropy subgroup of $\mathscr{P} \Omega G \times U(1)$ at any point of $\hat{Q}$ is the normal subgroup $K$ whose quotient is $\widehat{\Omega G}$ so that $\widehat{\Omega G}$ acts on $\hat{Q}$.

3. The orbits of the free action of $\widehat{\Omega G}$ on $\hat{Q}$ are the fibres of the projection $\hat{Q} \rightarrow M$ which sends a path in $Q$ to $p$ of its endpoint.

4. The action of $\widehat{\Omega G}$ on $\widehat{Q}$ covers the action of $\widehat{\Omega G}$ on $Q$,

It follows that $\hat{Q}$ is the desired lift of the bundle $Q$ to an $\widehat{\Omega G}$ bundle.

Now we will prove the converse of this result, that is, if $Q$ has a lift then there is a two form $\mu$ with $d \mu=f^{*}(\omega)$. We have to show that we can find a curvature form $2 \pi i F$ for the bundle $\hat{Q} \rightarrow Q$ that is both $\Omega G$ invariant and agrees with $2 \pi i{ }^{*} c$ in the directions tangent to the fibers of $p$. Assume that this is possible for now. Then $f^{*}(c)-F$ has the properties that:

i) it is zero on directions tangent to the fibres of $p$ and;

ii) the exterior derivative of it is $p^{*} f^{*}(\omega)$ which is zero on directions tangent to the fibres of $p$.

It can be readily checked in local co-ordinates that these conditions imply that $f^{*}(c)-F$ is pulled back from some two form on the base say $\mu$. So we have

$$
p^{*} d \mu=d\left(f^{*}(c)-F\right)=p^{*} f^{*}(\omega)
$$

and therefore $d \mu=f^{*}(\omega)$ as required. 
The remainder of this section is devoted to proving the existence of such an $F$. The reader willing to take this on faith may continue directly to Sect. 5 .

We first prove a simple result about central extensions of Lie algebras. Let

$$
0 \rightarrow \mathbf{R} \stackrel{\alpha}{\longrightarrow} \hat{M} \stackrel{\alpha}{\longrightarrow} M \rightarrow 0
$$

be a central extension of Lie algebras. We are interested in the case where $\hat{M}$ is isomorphic to the Kac-Moody algebra and $M$ is isomorphic to the loop algebra; but what we have to say here is quite general. Given such a central extension we can choose a splitting $\sigma: M \rightarrow \hat{M}$, that is a linear map with $\beta \circ \sigma$ equal to the identity and define a cocycle $c$ by

$$
c(X, Y)=\sigma([X, Y])-[\sigma(X), \sigma(Y)]
$$

for all $X$ and $Y$ in $M$. If $\sigma^{\prime}$ is another splitting then we have $\sigma-\sigma^{\prime}=\alpha \circ \gamma$ for some $\gamma: M \rightarrow \mathbf{R}$ and

$$
c(X, Y)-c^{\prime}(X, Y)=\gamma([X, Y]) .
$$

It follows that if two splittings give rise to the same cocycle then we must have $\gamma([X, Y])=0$ for all $X, Y$ in $M$ and therefore if $M$ is spanned by all such commutators (as the loop algebra is) that the splittings are the same. It follows that the cocycle determines the splitting.

Consider now a $U(1)$ bundle $P \rightarrow Y$, where $\widehat{\Omega G}$ acts freely and transitively on $P$ covering a free transitive action of $\Omega G$ on $Y$. Then we claim that for any $\Omega G$ invariant two form on $Y$ representing the Chern class of the bundle there is a unique $\widehat{\Omega G}$ invariant connection on $P$ whose curvature is $2 \pi i$ times that two form. Certainly there are invariant connections with this property; just fix a basepoint and identify everything with $\widehat{\Omega G} \rightarrow \Omega G$. Then the uniqueness follows by realising that the $\widehat{\Omega G}$ invariant vector fields on $P$ and the $\Omega G$ invariant vector fields on $Y$ are copies of the Kac-Moody algebra and the loop algebra respectively. The projection map sends invariant vector fields on $P$ to those on $Y$ and the invariant two form on $Y$ defines a cocycle. Now we can use the result above to show the uniqueness of the invariant connection.

From the fibering $\hat{Q} \rightarrow Q$ we can define a map of vector bundles

$$
T \hat{Q} / U(1) \rightarrow T Q
$$

over $Q$ and a connection is a splitting of this map (Atiyah (1957), pp. 187-188). If we factor further we obtain another map

$$
T \hat{Q} / \widehat{\Omega G} \rightarrow T Q / \Omega G
$$

of vector bundles over $X$. An $\Omega G$ invariant connection on $\hat{Q} \rightarrow Q$ is a splitting of this map. Inside each of the bundles in (4.16) we can consider the vectors tangent to the fibres of $\hat{p}: \hat{Q} \rightarrow X$ and $p$. These subbundles are, in fact, the adjoint bundles of Lie algebras,

$$
\operatorname{ad} \hat{Q} \rightarrow \operatorname{ad} Q \text {. }
$$

The map in (4.16) restricted to these subbundles defines, on fibres, a mapping between a Lie algebra isomorphic to the Kac-Moody algebra and a Lie algebra isomorphic to the loop algebra. Moreover the two form $f^{*}(c)$ defines a cocycle on each of these Lie algebras as it is an $\Omega G$ invariant form on each fibre of $Q \rightarrow X$. 
On each fibre above some $x \in X$, this is the situation we were in above with $P=\hat{p}^{-1}(x)$ and $Y=p^{-1}(x)$. It follows from our previous discussion that there is a splitting of (4.17) determined by $f^{*}(c)$. This can be extended to a splitting of (4.16) and this defines an $\widehat{\Omega G}$ invariant connection on $\hat{Q} \rightarrow Q$. Because we have extended from a splitting determined by $f^{*}(c)$ the curvature of the connection agrees with $2 \pi i f^{*}(c)$ on each fibre of $p$ as required.

\section{Explicit Formulae for the Loop Bundle}

We want to conclude with an explicit calculation of the pullback of the three form $\omega$ on $G$ by the classifying map hol: $\Omega M \rightarrow G$.

Consider the evaluation maps

and

$$
\mathrm{ev}_{P}: S^{1} \times \Omega P \rightarrow P
$$

$$
\mathrm{ev}_{P}:[0,1] \times \Omega P \rightarrow P,
$$

and similarly for $P$ replaced by $M$. Here of course we are regarding the circle $S^{1}$ as the interval $[0,1]$ with endpoints identified, hence the abuse of notation. We can pullback the bundle $P \rightarrow M$ with $\operatorname{ev}_{M}$ to the space $[0,1] \times M$ and then it is trivial. In fact a section is given by

$$
\begin{aligned}
h:[0,1] \times \Omega M & \rightarrow \operatorname{ev}_{M}^{*} P, \\
(t, \gamma) & \mapsto \hat{\gamma}(t),
\end{aligned}
$$

where $\hat{\gamma}$ is the horizontal lift of $\gamma$ to $P$ beginning at $p_{0}$. Let

$$
\operatorname{Pont}(F)=\frac{1}{8 \pi^{2}}\langle F \wedge F\rangle
$$

denote the Pontrjagin form for the curvature of the $G$ bundle $P$. Here if $f, g$ are Lie algebra valued functions and $\alpha, \beta$ are forms we define $\langle f \otimes \alpha \wedge g \otimes \beta\rangle=$ $\langle f, g\rangle \alpha \wedge \beta$. If $l_{\partial / \partial t}$ denotes contraction with the vector field in the $[0,1]$ or $S^{1}$ direction we have

$$
\begin{aligned}
\int_{S^{1}} l_{\partial / \partial t} \operatorname{ev}_{M}^{*} \operatorname{Pont}(F) d t & =\int_{[0,1]} l_{\partial / \partial t} \operatorname{ev}_{M}^{*} \operatorname{Pont}(F) d t \\
& =\int_{[0,1]} l_{\partial / \partial t} \operatorname{Pont}\left(\operatorname{ev}_{M}^{*}(F)\right) d t .
\end{aligned}
$$

If $A$ is the connection form on $P$ we can pull it back to $\operatorname{ev}^{*}(P)$ and then back to $[0,1] \times \Omega M$ to obtain

$$
\hat{A}=h^{*} \operatorname{ev}_{P}^{*}(A)
$$

Because $[0,1] \times M$ is a product manifold we can invariantly decompose the differential forms on $[0,1] \times M$ into components with a $d t$ and components without a $d t$. Under this decomposition $\hat{A}$ has no $d t$ component and we decompose $\mathrm{ev}_{M}^{*}(F)$ as

$$
\mathrm{ev}_{M}^{*}(F)=-\frac{\partial}{\partial t}(\widehat{A}) \wedge d t+\hat{F}
$$


The reason for calling the component of $\operatorname{ev}_{M}^{*}(F)$ without a $d t \hat{F}$ is that if we regard $\hat{A}$, for fixed $t_{0}$, as a connection form on $\Omega M$ and compute its curvature we get $\hat{F}$ evaluated at $t_{0}$.

If one substitutes (5.7) into (5.5) and uses the definition of Pont (5.4) one obtains

$$
\int_{[0,1]} l_{\partial / \partial t} \operatorname{ev}_{M}^{*} \operatorname{Pont}(F) d t=2 \int_{[0,1]} l_{\partial / \partial t}\left\langle-\frac{\partial}{\partial t}(\hat{A}) \wedge d t \wedge \hat{F}\right\rangle
$$

Expanding $\hat{F}=d \hat{A}+[\hat{A}, \hat{A}]$, where this $d$ is only acting in the $\Omega G$ directions one finds that $(5.8)$ is a sum of two terms;

and

$$
2 \int_{[0,1]}\left\langle-\frac{\partial}{\partial t}(\hat{A}) \wedge d \hat{A}\right\rangle
$$

$$
2 \underset{[0,1]}{\int}\left\langle-\frac{\partial}{\partial t}(\hat{A}) \wedge[\hat{A}, \hat{A}]\right\rangle \text {. }
$$

The form $\langle$,$\rangle is invariant so satisfies \langle X,[Y, Z]\rangle=\langle[X, Y], Z\rangle$ and applying this to the second term (5.10) and integrating by parts gives

$$
\frac{3}{2}\left\langle(d \mathrm{hol}) \mathrm{hol}^{-1} \wedge\left[(d \mathrm{hol}) \mathrm{hol}^{-1},(d \mathrm{hol}) \mathrm{hol}^{-1}\right]\right\rangle .
$$

Here this is hol: $\Omega M \rightarrow G$ and we use the facts that $\hat{A}(0)=$,0 and $h(0, \gamma)=$ $h(1, \gamma) \operatorname{hol}(\gamma)$ so that

$$
\hat{A}(0, \quad)=\operatorname{ad}\left(\mathrm{hol}^{-1}\right) \hat{A}(1, \quad)+\text { hol }^{-1} d \text { hol, }
$$

and therefore

$$
\hat{A}(1, \quad)=-(d \text { hol }) \mathrm{hol}^{-1} \text {. }
$$

For the other term (5.9) integration by parts in both the $\Omega M$ and $[0,1]$ directions gives

$$
-d \int_{[0,1]}\left\langle\frac{\partial}{\partial t}(\hat{A}) \wedge \hat{A}\right\rangle-\frac{1}{3}\left\langle(d \mathrm{hol}) \mathrm{hol}^{-1} \wedge\left[(d \mathrm{hol}) \mathrm{hol}^{-1},\left(d \mathrm{hol}^{-1} \mathrm{hol}^{-1}\right]\right\rangle .\right.
$$

Finally we recall the definition of $\omega$ (3.7) and $c(4.3)$ to obtain

$$
\operatorname{hol}^{*}(\omega)=\int_{S^{1}} l_{\partial / \partial t} \operatorname{ev}_{M}^{*} \operatorname{Pont}(F) d t+d c\left(h^{*} A, h^{*} A\right) .
$$

This shows that the string form is cohomologous to the integral over the circle of the pullback by the evaluation map of the Pontrjagin class. These formulae should be compared with those in Coquereaux and Pilch (1989).

\section{Appendix. Fréchet Manifolds}

We follow Hamilton (1982) for the theory of Fréchet manifolds.

If $M$ is a manifold then $\Omega M$ is a Fréchet manifold as follows. Regard functions from $S^{1}$ into $M$ instead as sections of the trivial bundle $S^{1} \times M$. The point of doing this is that sections are imbeddings, that is, they are injective with injective derivative, so thier image is a submanifold. Let $\gamma: S^{1} \rightarrow M$ be such a map and 
$\Gamma: S^{1} \rightarrow M \times S^{1}$ be the induced section of the trivial bundle. Let $T_{v}$ be the vector bundle over $M \times S^{1}$ which consists of the tangents to the vertical $(M)$ directions. Then there is a natural identification of $\gamma^{-1} T M$ and $\Gamma^{-1} T_{v}$. We can choose a tubular neighbourhood of the image of $\Gamma$ and identify this with an open neighbourhood of the zero section in $\Gamma^{-1} T_{v}$. The sections that lie in this tubular neighbourhood are thereby identified with sections of $\Gamma^{-1}\left(T_{v}\right)$. The space of all sections of $\Gamma^{-1}\left(T_{v}\right)$ forms a Fréchet space with the smooth topology and the set of sections that lie in an open neighbourhood of the zero section form an open set in this topology. This construction therefore defines co-ordinate charts on $\Omega M$. In Hamilton (1982) it is shown that these co-ordinate charts make $\Omega M$ a Fréchet manifold. Another result, also in Hamilton (1982) (p.91, Example 4.4.5), that we shall use repeatedly is that if $M$ is compact and $\phi: M \rightarrow N$ is a smooth map then the induced map $\Omega M \rightarrow \Omega N, \gamma \mapsto \phi \circ \gamma$ is a smooth map of Fréchet manifolds. We also use Hamilton's definition of a Fréchet principal bundle (Hamilton (1982) Definition 4.6.5, p.98).

Consider now the path fibration

$$
P G \rightarrow G
$$

of (3.1). The projection map here is evaluation at the endpoint which is continuous in the Fréchet topology and the action of $\Omega G$ on $P G$ is also continuous. We want to show then that this is a locally trivial $\Omega G$ bundle. We claim first that if $B$ is an open ball about the origin in Euclidean space of the same dimension as $G$ then for any $g$ in $G$ there is a smooth map $\phi:[0,1] \times B \rightarrow G$ which has the following properties. For $t \in[0,1 / 3)$ the map takes the constant value the identity in $G$, for $t \in(2 / 3,1]$ the map $\phi(t):, B \rightarrow G$ is a diffeomorphism onto its image and $\phi(1,0)=g$. Indeed if we replace $G$ by any ball in Euclidean space, and the identity and $g$ by points in this ball such maps can be constructed. For the general case just take a ball around $g$ and join the edge of it to the identity by a curve and then patch in one of the functions from the open ball case. Let us denote by $U$ the open ball about $g$ which is the image of $\phi(1$,$) and by V$ the pre-image of this under the projection $P G \rightarrow G$. Note then that the map $\phi$ defines a section $s$ over $U$, where $h=\phi(1, b)$ in $U$ gets mapped to the path $s(h)=\phi(, b)$. From this section we define a local trivialisation by

$$
\begin{aligned}
U \times \Omega G & \rightarrow V, \\
(h, x) & \rightarrow s(h) x .
\end{aligned}
$$

Notice that the theorems of Hamilton (1982) cited above show that this is a smooth map of Fréchet manifolds. The inverse of this map is defined as follows. Take $y$ in $V \subset P G$, then it is a path ending in $U$, at $h$ say. So also is the path $s(y)$, so the path $s(y)^{-1} y$ is a loop. Then $y$ is the image of the pair $\left(s(y), s(y)^{-1} y\right)$ under the trivialisation (A.2). Again Hamilton's results give the required smoothness and it follows that this is a diffeomorphism so the bundle is locally trivial and hence a Fréchet principal bundle.

If $P(M, G)$ is a principal $G$ bundle then there is an obvious pointwise action of $\Omega G$ on $\Omega P$ with quotient $\Omega M$. By the results above $\Omega M, \Omega P$, and $\Omega G$ are smooth Fréchet manifolds, the projection map $\Omega P \rightarrow \Omega M$ is smooth and the action of $\Omega G$ on $\Omega P$ is smooth. To prove that $\Omega P(\Omega M, \Omega G)$ is a Fréchet principal bundle 
it remains to show local triviality which we do as follows. As above we want to work with sections rather than maps, so we consider the principal bundle $S^{1} \times P\left(S^{1} \times M, G\right)$. Then choose an element $\gamma$ in $\Omega M$ and an element $\hat{\gamma}$ in the fibre of $\Omega P$ above $\gamma$. So the image of $\gamma$ is a loop in $S^{1} \times M$ and the image of $\hat{\gamma}$ is a loop in the total space of the bundle $S^{1} \times P$ above it. So it defines a section of this $G$ bundle above the image of $\gamma$. Take a tubular neighbourhood $N$ of the image of $\gamma$ then the bundle $S^{1} \times G$ restricted to this is trivial and we can extend the section defined by $\hat{\gamma}$ to a section $s$ over the whole of $N$. Now we have the corresponding open set $U$ in $\Omega M$ which is the neighbourhood of $\gamma$ consisting of all loops in $N$. Let $V$ be the open set in $\Omega P$ of all loops in $\pi^{-1}(U)$. Define a map

$$
\begin{aligned}
\Omega G \times U & \rightarrow V, \\
(g, \alpha) & \rightarrow\left(s^{\circ} \alpha\right) g .
\end{aligned}
$$

The results of Hamilton (1982) show that this map is smooth. It is clear that it has an explicit inverse that is also smooth. Again this follows from Hamilton's work.

Acknowledgements. The first author thanks Professors Michael Barber and Rodney Baxter for the opportunity to participate in the special program on Yang-Baxter equations at the Centre for Mathematical Analysis, Australian National University in 1989, and thanks the Centre for financial support. We also thank Roger Richardson for useful conversations and the referee for helpful comments.

\section{References}

Atiyah, M. F.: Complex analytic connections in fibre bundles. Trans. Am. Math. Soc. 85, 181-207 (1957)

Coquereaux, R., Pilch, K.: String structures on loop bundles. Commun. Math. Phys. 120, 353-378 (1989)

Hamilton, R. S.: The inverse function theorem of Nash and Moser. Bull Am. Math. Soc. 7 (1), 65-22 (1982)

Husemoller, D.: Fibre Bundles. Graduate Texts in Mathematics, vol. 20. Berlin, Heidelberg, New York: Springer 1966

Killingback, T.: World-sheet anomalies and loop geometry. Nucl. Phys. B288, 578-588 (1987)

Murray, M. K.: Another construction of the central extension of the loop group. Commun. Math. Phys. 116, 73-80 (1988)

Pressley, A., Segal, G.: Loop groups. Oxford Mathematical Monographs. London: Clarendon Press 1986

Singer, I. M.: Some remarks on the Gribov ambiguity. Commun. Math. Phys. 60, 7-12 (1978)

Communicated by A. Jaffe 